\title{
The m6A Methylation Gene SNRPC can be used as a Biomarker for the Prognosis and Immunotherapy of Hepatocellular Carcinoma
}

Jihao Cai ( $\square$ caijh@email.ncu.edu.cn )

Nanchang University Medical College: Medical College of Nanchang University https://orcid.org/00000002-8935-8708

\section{Minglei Zhou}

Shandong University of Technology - West Campus: Shandong University of Technology

Jianxin Xu

Nanchang University Medical College: Medical College of Nanchang University

\section{Research}

Keywords: SNRPC, hepatocellular carcinoma, N6-methyladenosine (m6A) RNA methylation, prognosis, immune checkpoint

Posted Date: March 22nd, 2021

DOI: https://doi.org/10.21203/rs.3.rs-320369/v1

License: (c) (1) This work is licensed under a Creative Commons Attribution 4.0 International License. Read Full License 


\section{Abstract}

Background: Hepatocellular carcinoma (HCC) is one of the most common malignancies in the world. Due to its complex pathogenic factors, the prognosis of HCC is poor. Therefore, a credible prognostic biomarker is urgently needed for this disease. N6-methyladenosine $\left(\mathrm{m}^{6} \mathrm{~A}\right)$ RNA methylation plays an important role in the tumorigenesis, progression and prognosis of many tumors. However, studies on the prognostic and therapeutic value of this modification in HCC are lacking.

Case Presentation: The HCC RNA-seq profiles in The Cancer Genome Atlas (TCGA) and International Cancer Genome Consortium (ICGC) databases, including 421 LIHC and 440 LIRI samples respectively, were used in this study. The expressive distinction of 21 RNA methylation regulators between HCC and normal tissue were firstly assessed and SNRPC was obtained. Then the expression of SNRPC was validated as a risk factor for prognosis by Kaplan-Meier analysis and employed to establish a nomogram with T pathologic stage. By GSVA and GSEA analyses, we found SNRPC was mainly related to protein metabolism and immune process. Further, ESTIMATE, MCP-counter and single sample GSEA (sSGSEA) algorithm showed high-SNRPC expression group had lower stromal scores, a lower abundance of endothelial cells, fibroblasts and immune infiltration. Ultimately, Tumor Immune Dysfunction and Exclusion (TIDE) analysis exhibited high-SNRPC expression group showed non-response to immune checkpoint inhibitor therapy, especially to a PD-1 inhibitor.

Conclusion: SNRPC could serve as valuable prognostic and immunotherapeutic marker in HCC. We provide here an accurate nomogram for clinical diagnosis using SNRPC as a biomarker.

\section{Background}

Primary liver cancer was the third leading cause of cancer death worldwide in 2020. Hepatocellular carcinoma (HCC) is the predominant form of primary liver cancer, which constitutes $75-85 \%$ of cases. The main risk factors for HCC include chronic infection with hepatitis B virus (HBV) or hepatitis $\mathrm{C}$ virus (HCV), heavy alcohol intake, type 2 diabetes and smoking[1]. According to the annual forecast of the World Health Organization, more than 1 million liver cancer patients will die by 2030 [2]. Fortunately, there are many treatments for liver cancer, such as liver transplantation, surgical resection, chemotherapy, radiotherapy, interventional therapy and immunotherapy. Surgical resection of early HCC is still the preferred treatment, but the recurrence rate at five years can be as high as 70\% [3]. Liver transplantation reduces the recurrence rate, but the number of available donor organs is always insufficient relative to the demand[4]. By contrast, the majority ( $>70 \%$ ) of patients with advanced disease do not benefit from surgery[5]. For these patients, immunotherapy is a potential option. In recent years, emerging immunotherapies, such as immune checkpoint blockade and CAR-T cell therapies, have shown better therapeutic outcomes on some tumors[6, 7]. Unfortunately, the responses to immunotherapy typically occur in a minority of patients, which range from $20-50 \%$ depending on the tumor type[8]. Thus, it is especially important to identify candidate biomarkers to target patients who have the greatest likelihood of being benefited[8]. In 2017, the Food and Drug Administration (FDA) gave an approval to the PD-1 
inhibitor nivolumab (OPDIVO $®$ ), which marked the start of the era of immunotherapy for HCC[9]. Compared with some tumors, immunotherapy for liver cancer is still in its infancy. Additionally, a good knowledge of the biomarkers for predicting the responses or primary resistance to immunotherapeutic agents is crucial for establishing immunotherapy criterion for HCC patients in the future.

The genome-wide analysis of mRNA expression profiles has been used to screen prognostic and treatment-related markers in many cancers in recent years [10-13]. The N6-methyladenosine (m6A) methylation modification occurs at the 6th $\mathrm{N}$ atom of adenine, is the most common post-transcriptional modification on RNA and mediates $>60 \%$ of RNA methylation $[14,15]$. Studies have elucidated that $\mathrm{m} 6 \mathrm{~A}$ RNA methylation plays an important role in tumor occurrence, development and prognosis[16]. However, the interaction between m6A methylation regulators and immunotherapy in HCC have not been reported to our knowledge. Therefore, in this study we aimed to analyze the potential of m6A RNA methylation regulators as prognostic biomarkers and predictors of immunotherapy responses of $\mathrm{HCC}$, so as to provide new references for the clinical treatment of HCC.

\section{Materials And Methods}

\subsection{Public transcriptome profiling}

In this study, we used $861 \mathrm{HCC}$ cases from two public databases. After excluding samples without clinical annotation and survival information, there were $421 \mathrm{LIHC}$ samples (50 normal samples labeled with 11A, and 364 HCC samples labeled with 01A) from The Cancer Genome Atlas (TCGA) database, which we used as training set. Additionally, 440 LIRI cases ( 197 normal and $243 \mathrm{HCC}$ ) from the International Cancer Genome Consortium (ICGC) database were used as a validation set.

\subsection{Identification of differential expression m6A-methylase genes (DEMGs)}

The limma package in R [17] was used to screen differential expression genes (DEGs) between normal and HCC groups using TCGA dataset. $\left|\log _{2}(\mathrm{FC})\right|>1$ and a $p$ value $<0.05$ were applied as screening thresholds. The DEGs identified are displayed using volcano plot and heatmap[18, 19]. Then the DEGs were intersected with 21 m6A methylation genes by Venn analysis. Finally, we obtained a set of differential expression m6A genes (DEMGs). Wilcoxon test was used to verify the significance difference in expression levels of DEMGs in TCGA and ICGC datasets.

\subsection{Survival analysis of DEMGs}

To evaluate if the identified DEMGs could impact survival probability of HCC, all of the HCC samples in TCGA and ICGC databases were divided into high- or low- expression groups, based on the expression median value of each DEMG. Survival probabilities were analyzed by Kaplan-Meier analysis and log-rank test [20], with a $p$ value $<0.05$ being considered statistically significant.

\subsection{Construction of a nomogram for prognostic prediction}


We established a nomogram by univariate and multivariate Cox regression analysis to evaluate the probability of 1-, 3- and 5-year overall survival for HCC patients via the rms R package [21] by integrating clinical factors and DEMG expression values.

\subsection{Functional annotation}

To investigate the potential biological mechanisms of DEMGs in HCC, all of the differentially expressed genes between high- or low- DEMG expression groups of HCC in TCGA database were ranked based on $\log _{2} \mathrm{FC}$ values. Then gene set enrichment analysis (GSEA) was implemented using the clusterProfiler package [22] to estimate the pathway activity changes in the above two groups. Similarly, The Gene Set Variation (GSVA) package[23] was utilized to find the predominate pathways associated with these DEMGs. Terms with an FDR $<0.25$ and $p<0.05$ were retained.

\subsection{ESTIMATE analysis}

The ESTIMATE algorithm can calculate immune scores and stromal scores via the estimate package in $\mathrm{R}[24]$, allowing one to quantify the immune and stromal components in a tumor. The difference of immune scores, stromal scores and tumor purity in high- or low-DEMG expression groups of HCC in TCGA database was assessed by Wilcoxon test.

\subsection{Analysis of tumor microenvironment}

To gain insight into the tumor microenvironment of HCC in TCGA database, MCP-counter[25] was used to quantify the numbers of immune cells, fibroblasts and epithelial cells from each HCC sample based on marker genes. Then, the ssGSEA algorithm was used to calculate the immune infiltration of each sample, and significant differences in immune cell numbers were identified by ANOVA.

\subsection{Tumor Immune Dysfunction and Exclusion (TIDE) analysis}

Immune checkpoint pathways are associated with tumor immune escape. Therefore, immune checkpoint inhibitors can enhance anti-cancer immunity. We employed the Tumor Immune Dysfunction and Exclusion (TIDE) algorithm[26] and subclass mapping[27] to predict the clinical response to immune checkpoints inhibitors in our high- and low-DEMGs expression groups.

\subsection{Statistical analysis}

All statistical tests were performed using $R$ (version 3.5.2) software. Continuous data were analyzed using the Wilcoxon test or ANOVA. A $p$ value of less than 0.05 was considered statistically significant in all analyses.

\section{Results}

\subsection{Identification of DEMGs}


We identified 1415 DEGs between normal and HCC samples, including 1015 up-regulated and 400 downregulated genes (Fig. 1A, B). Then, only SNRPC was identified as a DEMG in HCC from the intersection of DEGs and m6A methylation genes (Fig. 1C). In both TCGA and the ICGC datasets, the expression levels of $S N R P C$ in tumors were always higher than normal samples (Fig. 1D, E).

\subsection{SNRPC is related to survival of HCC}

A Kaplan-Meier survival curve was drawn, and the results showed that both in TCGA and ICGC databases, the low-SNRPC expression group had a high survival probability relative to the high-SNRPC expression group $(p<0.05)$ (Fig. 2A, B). This suggested that SNRPC impacted outcome in HCC patients.

\subsection{Construction of a nomogram for HCC prognosis}

Univariate and multivariate Cox regression analyses results showed that T (pathological stage) and $S N R P C$ were significantly correlated with the prognosis of HCC (Fig. 2C, D). Therefore, a nomogram including T clinical factors and SNRPC expression values was constructed for predicting 1-, 3- and 5-year survival probabilities of HCC using samples from TCGA database (Fig. 2E). A calibration curve demonstrated that our nomogram could accurately estimate the mortality of HCC (Fig. 2F).

\subsection{Functional annotation}

To gain insight into the potential mechanisms related to $S N R P C$, we first selected 64 DEGs with differences between the high- and low-SNRPC expression groups (Fig. 3A). Then, we found that GO terms including catalytic_activity_acting_on_DNA, catalytic_step_2_spliceosome and catalytic_ activity_acting_on_RNA were activated, while terms such as acute_inflammatory_response, alcohol_metabolic_process, alpha_amino_acid_catabolic_process, alpha_amino_acid_metabolic_process, blood_microparticle, cellular_amino_acid_catabolic_process and cellular_lipid_catabolic_process, were suppressed in the high-SNRPC expression group (Fig. 3B). In addition, KEGG pathways such as ribosome, DNA_replication, spliceosome and cell_cycle was up-regulated, while PPAR_signaling_pathway, complement_and_coagulation_cascades, drug_metabolism_cytochrome_p450, fatty_acid_metabolism, retinol_metabolism and valine_leucine_and_isoleucine_degradation, were down-regulated in the highSNRPC expression group (Fig. 3C).

In addition, compared to the low-expression group, there were 19 immune-related pathways dysregulated in the high-SNRPC expression group. Among them,

ANTIGEN_PROCESSING_AND_PRESENTATION_OF_PEPTIDE_ANTIGEN, ANTIGEN_RECEPTOR_MEDIATED_SIGNALING_PATHWAY, ANTIGEN_PROCESSING_

AND_PRESENTATION, INNATE_IMMUNE_RESPONSE_ACTIVATING_CELL_

SURFACE_RECEPTOR_SIGNALING_PATHWAY, ANTIGEN_PROCESSING_ AND_PRESENTATION_OF_PEPTIDE_ANTIGEN_VIA_MHC_CLASS_I,

ANTIGEN_PROCESSING_AND_PRESENTATION_OF_EXOGENOUS_PEPTIDE_ANTIGEN_VIA_MHC_CLASS_I and SOMATIC_DIVERSIFICATION_OF_IMMUNE_

RECEPTORS_VIA_GERMLINE_RECOMBINATION_WITHIN_A_SINGLE_LOCUS were inhibited, while 
DENDRITIC_CELL_ANTIGEN_PROCESSING_AND_PRESENTATION, IMMUNOGLOBULIN_BINDING, REGULATION_OF_HUMORAL_IMMUNE_RESPONSE,

HUMORAL_IMMUNE_RESPONSE_MEDIATED_BY_CIRCULATING_IMMUNOGLOBULIN, B_CELL_MEDIATED_IMMUNITY, HUMORAL_IMMUNE_RESPONSE, LYMPHOCYTE_MEDIATED_IMMUNITY, ADAPTIVE_IMMUNE_RESPONSE, ADAPTIVE_IMMUNE_RESPONSE_BASED_ON_SOMATIC_RECOMBINATION_OF_

IMMUNE_RECEPTORS_BUILT_FROM_IMMUNOGLOBULIN_SUPERFAMILY_DOMAINS, REGULATION_OF_IMMUNE_EFFECTOR_PROCESS, IMMUNE_RECEPTOR_ACTIVITY and NEGATIVE_REGULATION_OF_IMMUNE_SYSTEM_PROCESS were enhanced in the high-SNRPC expression group (Fig. 3D, E). These results implied that SNRPC was correlated with the immune system.

\subsection{ESTIMATE analysis}

The cells included in the tumor microenvironment impact tumor progress. In our study, we found that except for immune scores, the stromal scores, ESTIMATE scores and tumor purity were significantly different between the high- and low-SNRPC expression groups (Fig. 4A-D). We also found that a lower stromal score and a higher tumor purity were associated with poor survival in HCC patients.

\subsection{Analysis of tumor microenvironment (TME)}

MCP-counter and SsGSEA analyses were conducted to clear the abundance of various cells in TME. Immune cells such as T cells, CD8 T cells and neutrophils and stromal cells, such as endothelial cells and fibroblasts, were significantly different between the high- and low-SNRPC expression groups. Among these cell types, neutrophils, endothelial cells and fibroblasts were increased in the low-expression group, while others were decreased (Fig. 5A, B). Through ssGSEA analysis, additional sub-types of immune cells were counted. In the low-SNRPC expression group, only activated CD4 T cells were significantly decreased, while CD56dim natural killer cells, central memory CD8 T cells, effector memory CD8 T cells, eosinophils, immature dendritic cells, macrophages, memory B cells, monocytes, natural killer T cells, natural killer T cells, plasmacytoid dendritic cells, regulatory $\mathrm{T}$ cells, type $1 \mathrm{~T}$ helper cells and type $17 \mathrm{~T}$ helper cells were all increased (Fig. 5C, D). These results implied that both stromal cells and immune cells were abundant and were beneficial for HCC prognosis.

\subsection{TIDE analysis}

TIDE score is used for predicting the efficacy of anti-PD1 and anti-CTLA4 treatments. The high-SNRPC expression group acquired a higher TIDE score using samples from both TCGA and ICGC databases, which suggested that they had poor efficacy in immune checkpoint inhibitor therapies (Fig. 6A, B). Consistently, the high-SNRPC expression group also showed non-response to PD1 therapy by subclass mapping analysis (Fig. 6B, 6C).

\section{Discussion}


HCC accounts for $75-85 \%$ of primary liver cancers and is the third leading cause of cancer-related death globally. Immunotherapy has become a viable option for advanced patients. However, not all patients show complete response to such immunotherapies[28]. Therefore, a reliable biomarker for predicting prognosis and immunotherapy response is urgently needed. Studies have shown that the regulators of m6A methylation have significant functions in cancer development, impacting processes such as proliferation, migration and invasion. Previous studies have indicated that some m6A RNA methylation regulators like YTHDF2, METTL3 and METTL14 were aberrantly expressed in HCC tumors, which may have been related to tumorigenicity, metastasis and poor prognosis[29]. In this study, we found an m6A RNA methylation regulator $S N R P C$, which was expressed differently in $\mathrm{HCC}$ compared with normal tissues. Studies have found that SNRP family genes play an important role in tumorigenesis and prognosis. For example, the high expression of SNRPG in different types of cancer is positively correlated with the occurrence, progression and severity of these diseases. The SNRPN gene is highly expressed in colorectal cancer tissues and is involved in the progression of this disease[30-32]. Also, compared with normal tissue, SNRPC was obviously overexpressed in HCC samples. Next, we divided our samples into two groups based on the expression of SNRPC. We found that the group with high-SNRPC expression showed lower survival and poorer immunotherapy response. Thus, SNRPC could act as a biomarker to predict both HCC prognosis and immunotherapy reaction.

RNA splicing is an essential process that governs many aspects of cellular proliferation, survival and differentiation. Alterations in this pathway have been implicated in many human cancers[33]. In this study, we found that the spliceosome and cell cycle components were up-regulated in the high-SNRPC expression group, which may have been related to its poor prognosis. In addition, we found that there were obvious defects in antigen processing and presentation pathways in the high-SNRPC expression group, which would have affected the effectiveness of immune checkpoint inhibitor (ICI) treatment[34]. Use of the TIDE algorithm also confirmed the above conclusion. Furthermore, TIDE score can also directly guide the use of ICls. Patients with higher TIDE score have higher anti-tumor immune escape opportunities, which results in lower ICls treatment response rates[26]. Moreover, the TIDE score has been shown to have a higher accuracy than PD-L1 expression level and TMB in predicting the survival outcome of cancer patients treated with ICB agents [35-37]. Consequently, the high-SNRPC expression group, with high its TIDE scores, had poor responses to ICI treatments.

$\mathrm{CD}^{+} \mathrm{T}$ cells bear the role of tumor immune surveillance and have direct killing functions on tumor cells. $\mathrm{CD}^{+} \mathrm{T}$ cells in HCC tissues have been shown to be significantly correlated with tumor recurrence and relapse-free survival (RFS)[38]. The low infiltration of $\mathrm{CD}^{+} \mathrm{T}$ cells is related to a variety of adverse prognostic factors. In our study, the TME analysis showed that the CD8 + T cell infiltration in the highSNRPC expression group was significantly lower than that in the low-SNRPC expression group, which may partially explain the poor overall survival (OS) in the high-SNRPC expression group. Previous studies have confirmed that the expression of PD-L1 was positively correlated with the density of CD8 + T cells[38], and samples with a high expression of PD-L1 had a higher response to ICIs[39]. In this study, the high-SNRPC expression group had lower CD $8+T$ cell infiltration, so we speculated that it had lower PD-L1 
expression. Through the TIDE analysis, we found that the high-SNRPC expression group showed no therapeutic response to ICls. Therefore, we speculated that SNRPC affected the therapeutic response of the PD-L1 inhibitor by affecting the infiltration of CD $8+T$ cells.

The limitation is that there is only a small repertoire of immunotherapies currently used for liver cancers, such as the immune checkpoint inhibitors nivolumab. As a result, we only test anti-PD1 and anti-CTLA4 treatments in our TIDE analyses rather than considering more promising immunotherapy methods such as CAR-T. At present, a large number of immune checkpoint inhibitors for HCC treatment intersect with PD-1; thus, our research can be used to guide immunotherapeutic decision-making in clinical settings.

\section{Conclusion}

In this study, we found a biomarker, SNRPC. in HCC, which not only predicted the prognosis of HCC but also functioned to indicate the efficacy of immunotherapy. SNRPC can help clinicians to target the patients who are most likely to benefit from immunotherapy and help them receive immunotherapies at earlier clinical stages. It is feasible to establish a clinical scoring system that contains SNRPC to predict HCC prognosis and immunotherapy response.

\section{Abbreviations}




\begin{tabular}{|ll|}
\hline HCC & Hepatocellular carcinoma \\
\hline $\mathrm{m}^{6} \mathrm{~A}$ & N6-methyladenosine \\
\hline TCGA & The Cancer Genome Atlas \\
\hline ICGC & International Cancer Genome Consortium \\
\hline DEMGs & Differentially Expressed m6A Methylation Genes \\
\hline GSEA & Gene Set Enrichment Analysis \\
\hline GSVA & Gene Set Variation Analysis \\
\hline SSGSEA & single sample GSEA \\
\hline TIDE & Tumor Immune Dysfunction and Exclusion \\
\hline HBV & Hepatitis B Virus \\
\hline HCV & Hepatitis C Virus \\
\hline FDA & Food and Drug Administration \\
\hline TME & Tumor Microenvironment \\
\hline ICl & Immune Checkpoint Inhibitor \\
\hline RFS & Relapse-free Survival \\
\hline OS & Overall Survival \\
\hline
\end{tabular}

\section{Declarations}

Ethics approval and consent to participate

Not applicable.

Consent for publication

Not applicable.

Availability of data and materials

The datasets analyzed during the current study are available in the TGCA, [https://portal.gdc.cancer.gov] and ICGC [https://dcc.icgc.org/] public database

Acknowledgements 
We thank LetPub (www.letpub.com) for its linguistic assistance during the preparation of this manuscript.

Competing interests

The authors declare that they have no competing interests.

Funding

This research did not receive any specific grant from funding agencies in the public, commercial, or notfor-profit sectors.

Authors' contribution

All authors were responsible for the analysis, interpretation of data, and graphing. Jihao Cai drafted the manuscript. All authors read and approved the final manuscript.

\section{References}

1. Sung $\mathrm{H}$, et al., Global cancer statistics 2020: GLOBOCAN estimates of incidence and mortality worldwide for 36 cancers in 185 countries. CA Cancer J Clin, 2021.

2. Karb DB, Sclair SN. Hepatocellular Carcinoma, in Liver Disease. 2019. p. 141-154.

3. Llovet JM, et al. Hepatocellular carcinoma. Nat Rev Dis Primers. 2016;2:16018.

4. Vivarelli $\mathrm{M}$, et al. Liver transplantation for hepatocellular carcinoma under calcineurin inhibitors: reassessment of risk factors for tumor recurrence. Ann Surg. 2008;248(5):857-62.

5. Thomas MB, et al. Hepatocellular carcinoma: consensus recommendations of the National Cancer Institute Clinical Trials Planning Meeting. J Clin Oncol. 2010;28(25):3994-4005.

6. Fournier $C$, et al. Trial Watch: Adoptively transferred cells for anticancer immunotherapy. Oncoimmunology. 2017;6(11):e1363139.

7. Smyth MJ, et al. Combination cancer immunotherapies tailored to the tumour microenvironment. Nat Rev Clin Oncol. 2016;13(3):143-58.

8. Llovet JM, et al. Molecular therapies and precision medicine for hepatocellular carcinoma. Nature Reviews Clinical Oncology. 2018;15(10):599-616.

9. Johnston MP, Khakoo SI. Immunotherapy for hepatocellular carcinoma: Current and future. World J Gastroenterol. 2019;25(24):2977-89.

10. Boyault S, et al. Transcriptome classification of HCC is related to gene alterations and to new therapeutic targets. Hepatology. 2007;45(1):42-52.

11. Chiang DY, et al. Focal gains of VEGFA and molecular classification of hepatocellular carcinoma. Cancer Res. 2008;68(16):6779-88. 
12. Hoshida $Y$, et al. Integrative transcriptome analysis reveals common molecular subclasses of human hepatocellular carcinoma. Cancer Res. 2009;69(18):7385-92.

13. Lee JS, et al. Classification and prediction of survival in hepatocellular carcinoma by gene expression profiling. Hepatology. 2004;40(3):667-76.

14. Du K, et al. m(6)A RNA Methylation Controls Neural Development and Is Involved in Human Diseases. Mol Neurobiol. 2019;56(3):1596-606.

15. Wang $\mathrm{S}$, et al. Roles of RNA methylation by means of $\mathrm{N}(6)$-methyladenosine $(\mathrm{m}(6) \mathrm{A})$ in human cancers. Cancer Lett. 2017;408:112-20.

16. Chen $\mathrm{J}$, et al. Identification of a m(6)A RNA methylation regulators-based signature for predicting the prognosis of clear cell renal carcinoma. Cancer Cell Int. 2020;20:157.

17. Smyth GK. Linear models and empirical bayes methods for assessing differential expression in microarray experiments. Stat Appl Genet Mol Biol. 2004;3:Article3.

18. Wickham H. ggplot2: elegant graphics for data analysis[M]. springer, 2016.

19. Raivo Kolde (2019). pheatmap: Pretty Heatmaps. R package version 1.0.12. https://CRAN.Rproject.org/package=pheatmap.

20. Heagerty PJ, Saha-Chaudhuri P, Saha-Chaudhuri M P. Package 'survivalROC' [J]. 2013.

21. Harrell JFE. Harrell Jr M F E, Hmisc D. Package 'rms'[J]. Vanderbilt University, 2019, 229.

22. $\mathrm{Yu} \mathrm{G}$, et al. clusterProfiler: an $\mathrm{R}$ package for comparing biological themes among gene clusters. OMICS. 2012;16(5):284-7.

23."nzelmann SH, Castelo R, Guinney J. GSVA: gene set variation analysis for microarray and RNA-Seq data. BMC Bioinformatics, 2013.

24. Kosuke, Yoshihara, Hoon Kim and Roel GW Verhaak (2016). estimate: Estimate of Stromal and Immune Cells in Malignant Tumor Tissues from Expression Data. R package version 1.0.13/r21..

25 . Becht $E$, et al. Estimating the population abundance of tissue-infiltrating immune and stromal cell populations using gene expression. Genome Biol. 2016;17(1):218.

26. Jiang $P$, et al. Signatures of $T$ cell dysfunction and exclusion predict cancer immunotherapy response. Nat Med. 2018;24(10):1550-8.

27. Hoshida $Y$, et al. Subclass mapping: identifying common subtypes in independent disease data sets. PLoS One. 2007;2(11):e1195.

28. Weber JS, et al. Toxicities of Immunotherapy for the Practitioner. J Clin Oncol. 2015;33(18):2092-9.

29. Liu ZX, et al. Link Between m6A Modification and Cancers. Front Bioeng Biotechnol. 2018;6:89.

30. Lloyd, Mabonga, Kappo AP. The oncogenic potential of small nuclear ribonucleoprotein polypeptide G: a comprehensive and perspective view. Am J Transl Res.

31. Ji M, et al., Small Nuclear Ribonucleoprotein Polypeptide N Accelerates Malignant Progression and Poor Prognosis in Colorectal Cancer Transcriptionally Regulated by E2F8. Frontiers in Oncology, 2020. 10. 
32. Yuan M, et al. Investigation on Potential Correlation Between Small Nuclear Ribonucleoprotein Polypeptide A and Lung Cancer. Front Genet. 2020;11:610704.

33. Wang E, Aifantis I. RNA Splicing and Cancer. Trends in Cancer. 2020;6(8):631-44.

34. Zaretsky JM, et al. Mutations Associated with Acquired Resistance to PD-1 Blockade in Melanoma. N Engl J Med. 2016;375(9):819-29.

35. Kaderbhai C, Tharin Z, Ghiringhelli F. The Role of Molecular Profiling to Predict the Response to Immune Checkpoint Inhibitors in Lung Cancer. Cancers (Basel), 2019. 11(2).

36. Keenan TE, Burke KP, Van Allen EM. Genomic correlates of response to immune checkpoint blockade. Nat Med. 2019;25(3):389-402.

37. Wang S, et al., Antigen presentation and tumor immunogenicity in cancer immunotherapy response prediction. Elife, 2019. 8.

38. Gabrielson A, et al. Intratumoral CD3 and CD8 T-cell Densities Associated with Relapse-Free Survival in HCC. Cancer Immunol Res. 2016;4(5):419-30.

39. Hilmi M, et al., Immune Therapy for Liver Cancers. Cancers (Basel), 2019. 12(1).

\section{Figures}




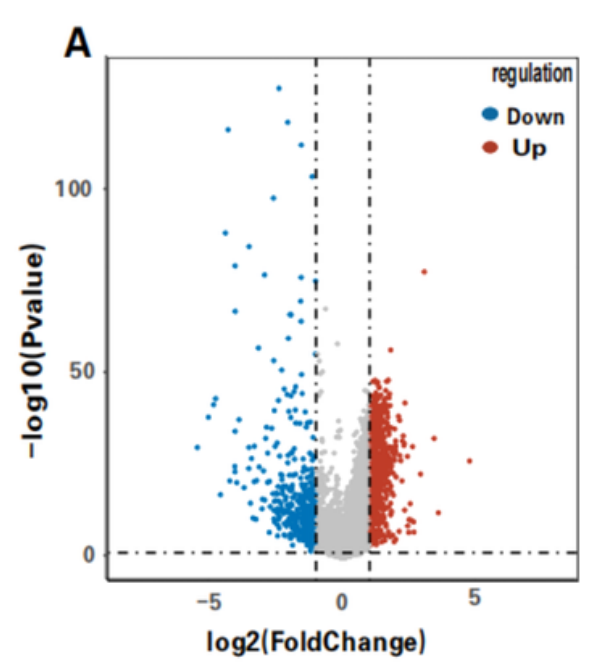

D

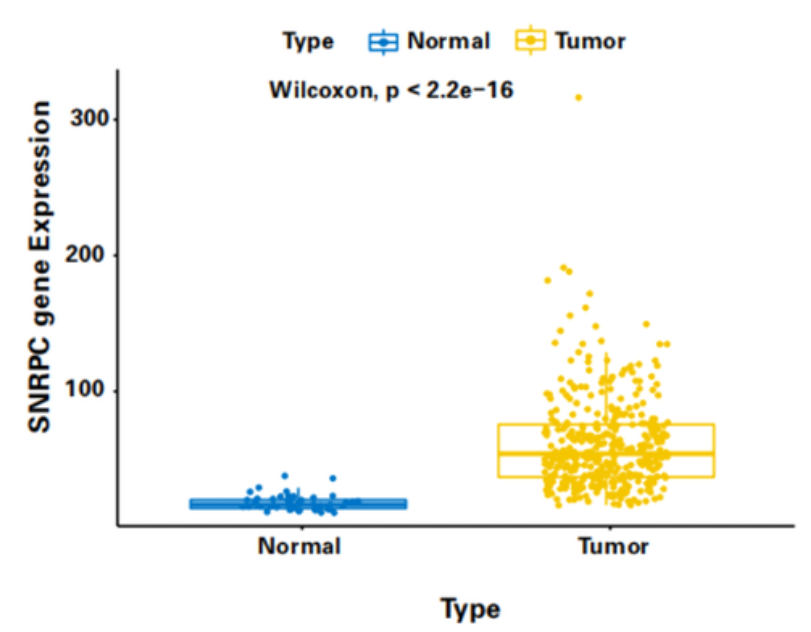

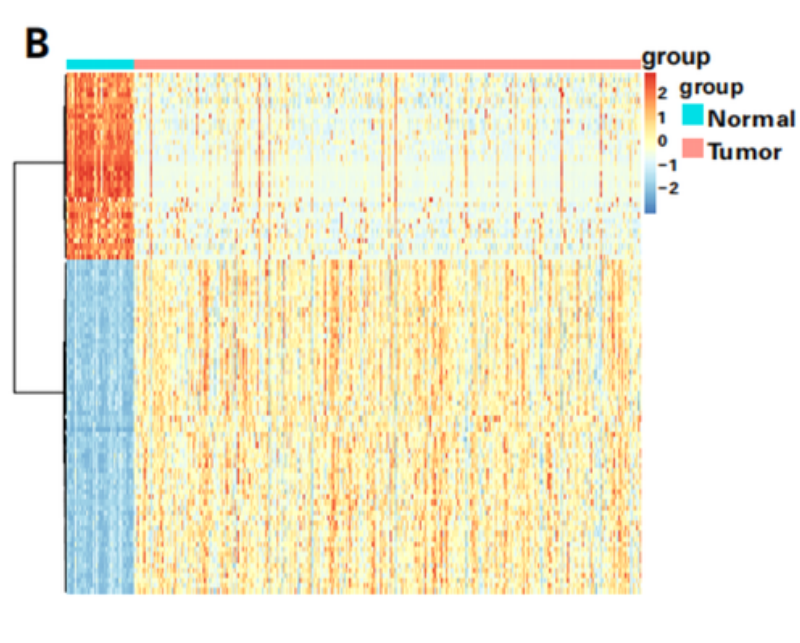

C

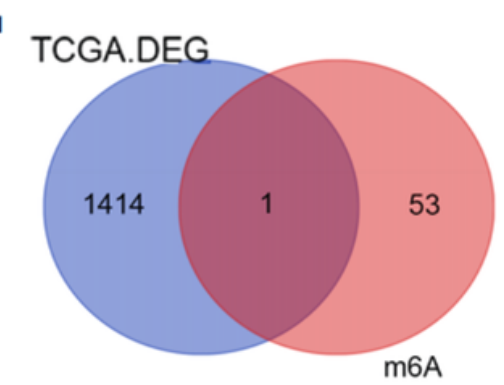

\section{Figure 1}

(A) Volcano map of gene expression in normal and HCC samples. (B) DEGs of normal and HCC tumor samples. (C) Venn diagram of DEMGs. (D) Expression of the SNRPC gene in TCGA-LIHC dataset. (E) Expression of the SNRPC gene in the ICGC-LIRI dataset. 


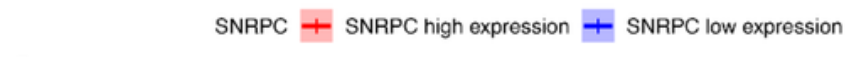

A

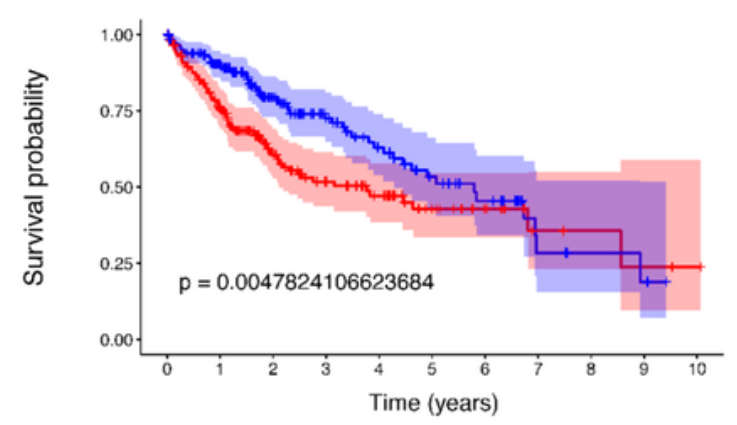

O. SNRPC high expression. $\begin{array}{lllllllllll}182 & 119 & 61 & 39 & 28 & 16 & 10 & 4 & 3 & 2 & 1\end{array}$

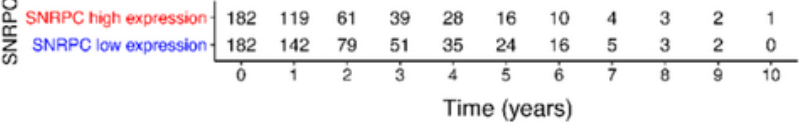

C

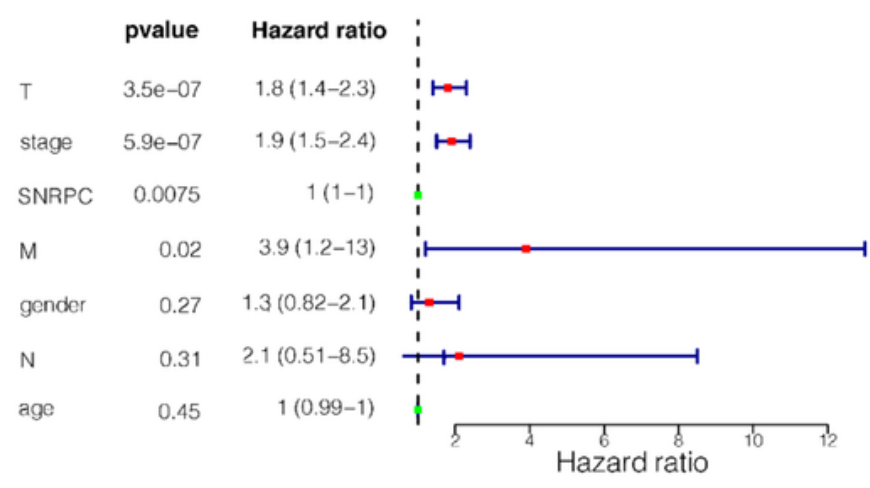

E

Points

T

SNRPC

Total Points

Linear Predictor

1-year survival Probability

3-year survival Probability

5-year survival Probability $0 . \overline{8}$
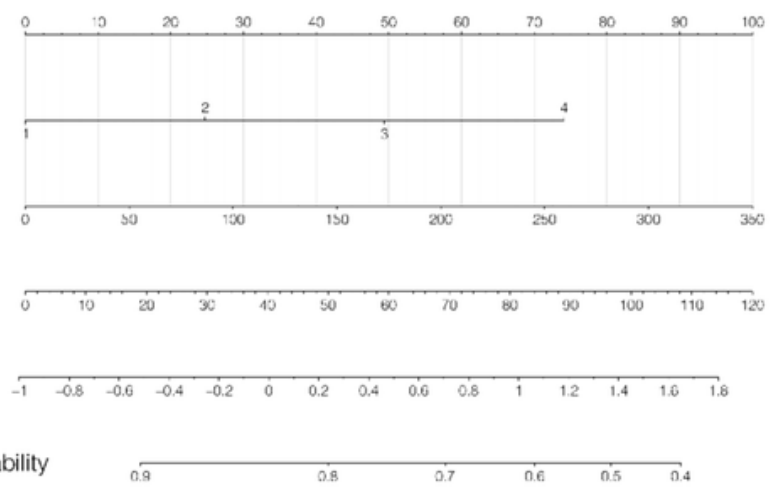

$\begin{array}{lllllll}0.8 & \text { i. } & \text { i. } & 0.4 & 0.3 & \end{array}$

$\begin{array}{lllllll}0.7 & 0.6 & 0.5 & 0.4 & 0.3 & 0.2 & 0.1\end{array}$
B

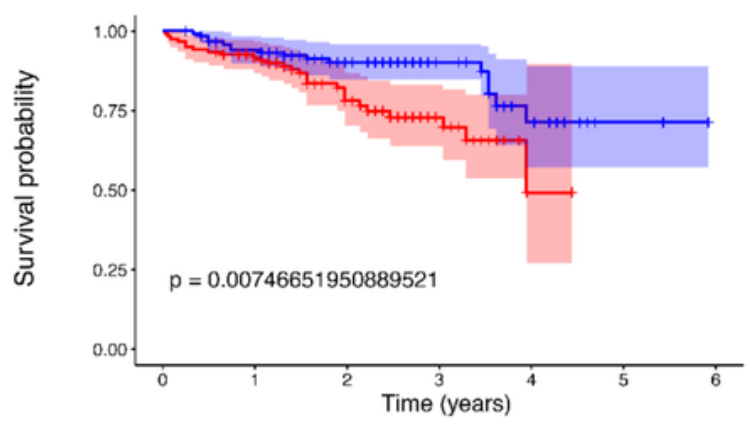

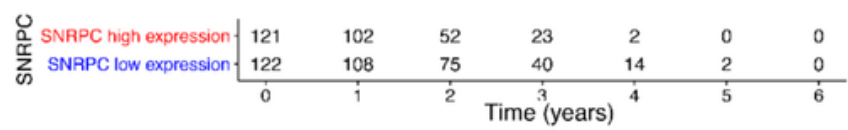

D

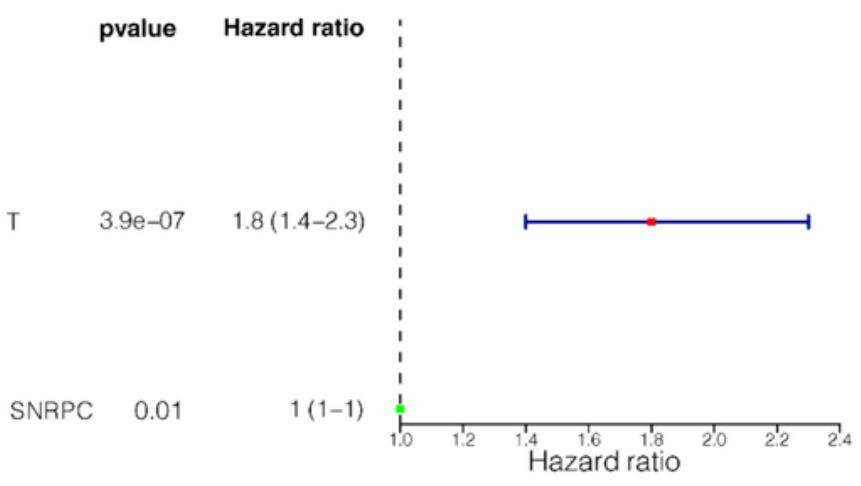

$\mathbf{F}$

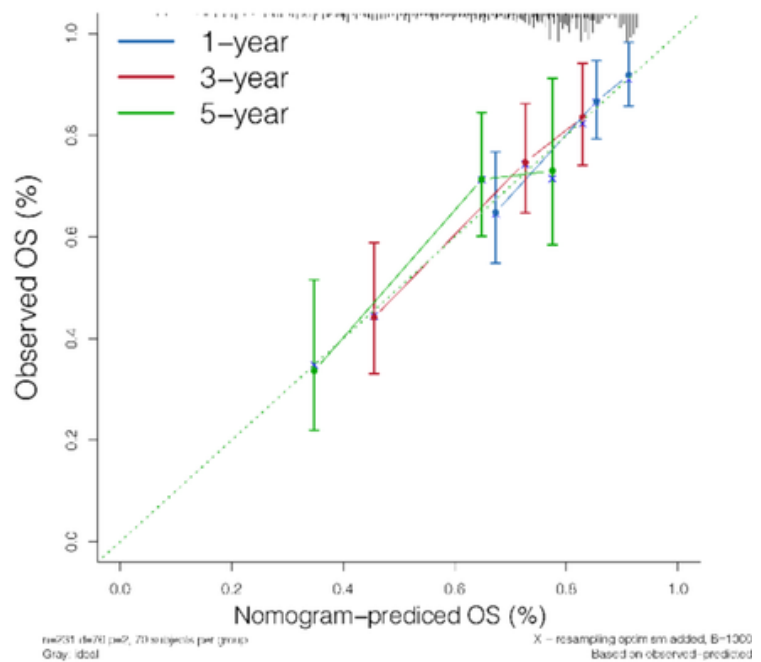

\section{Figure 2}

(A) KM survival curve of total survival time based on the SNRPC gene in TCGA-LIHC dataset. (B) KM survival curve of total survival time based on the SNRPC gene in the ICGC-LIRI dataset. (C) Univariate COX regression analysis. (D) Multivariate COX regression analysis. (E) Nomogram for HCC 1-, 3- and 5year prognosis. (F) Calibration curve. 

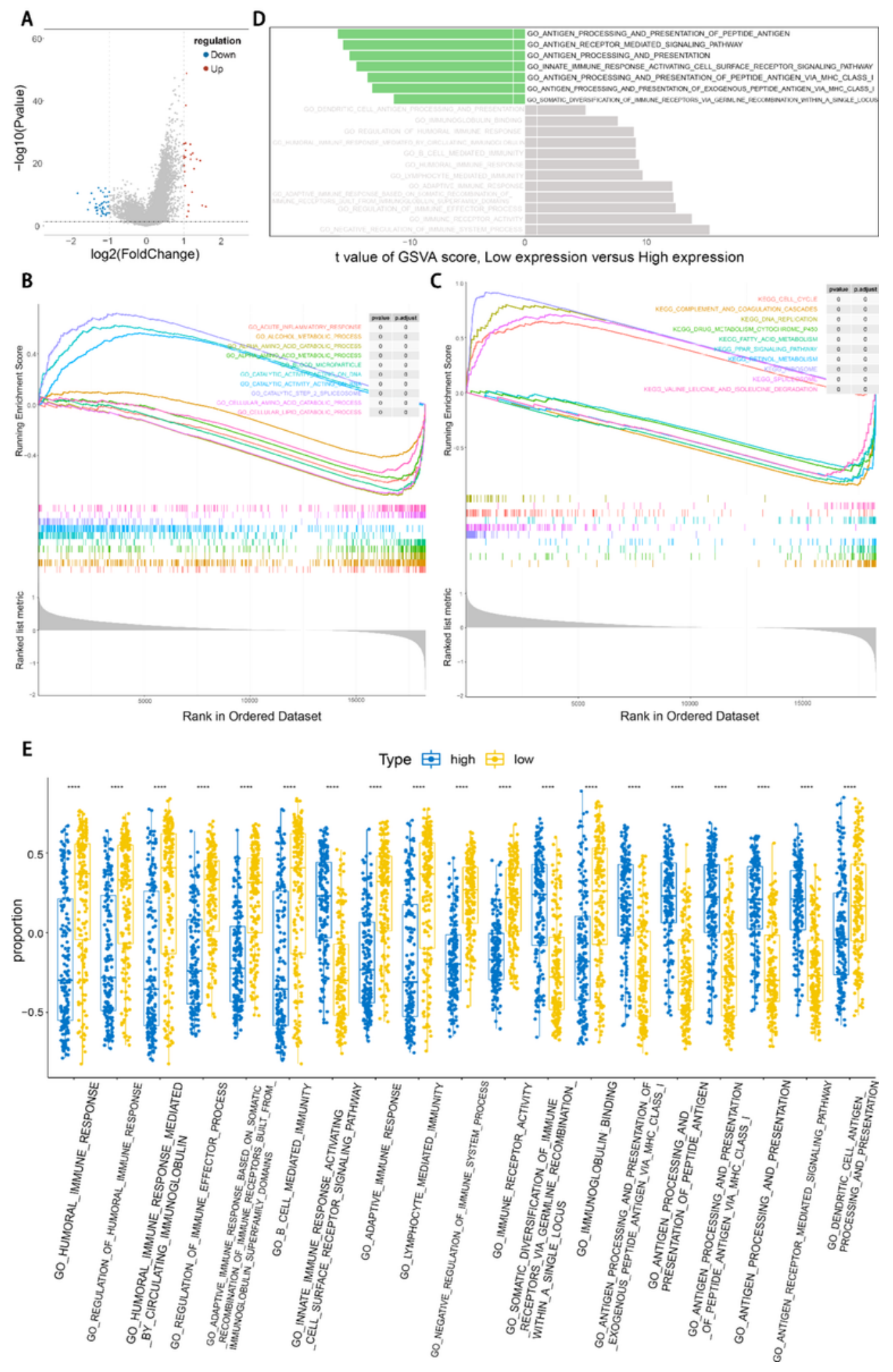

\section{Figure 3}

(A) Volcano map showing the low- and high-SNRPC expression groups. (B) Top $10 \mathrm{GO}$ terms annotated in the high-SNRPC expression group. (C) Top 10 KEGG terms enriched in the high-SNRPC expression group. (D, E) Nineteen immune-related items enriched by GSVA. 


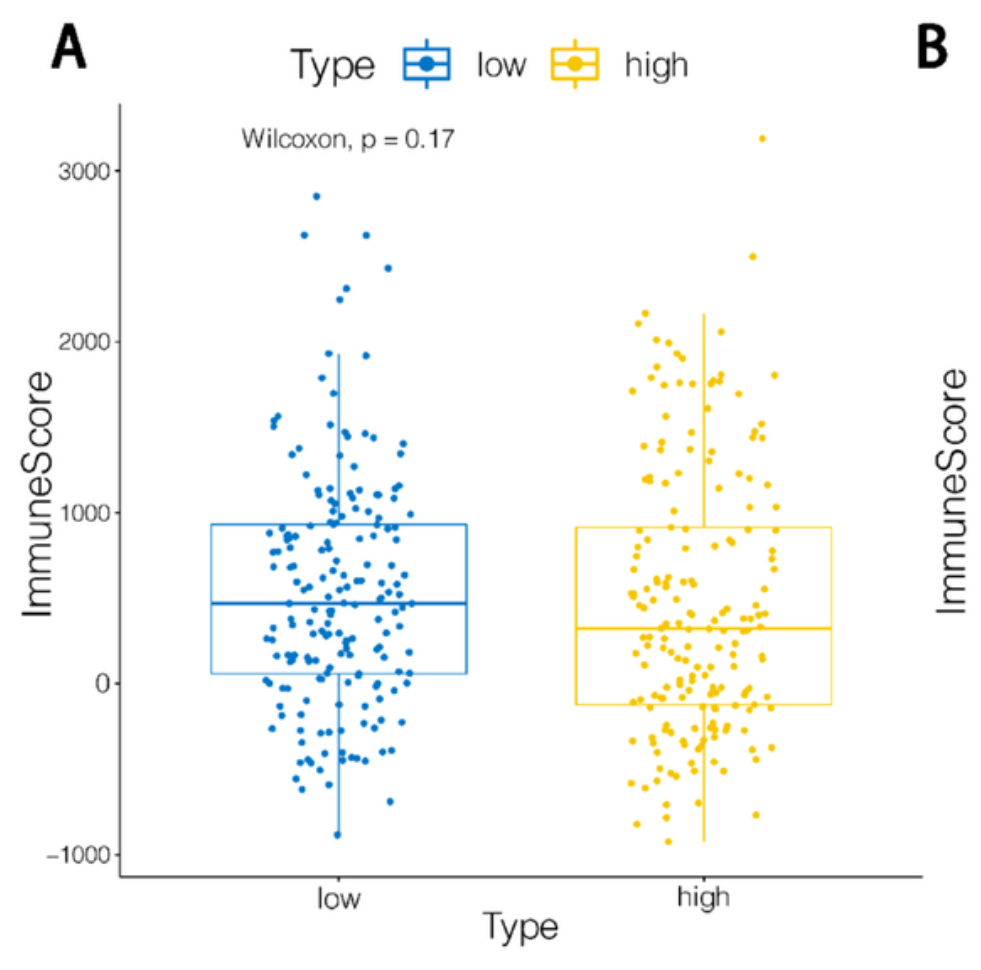

B Type 庐 low high
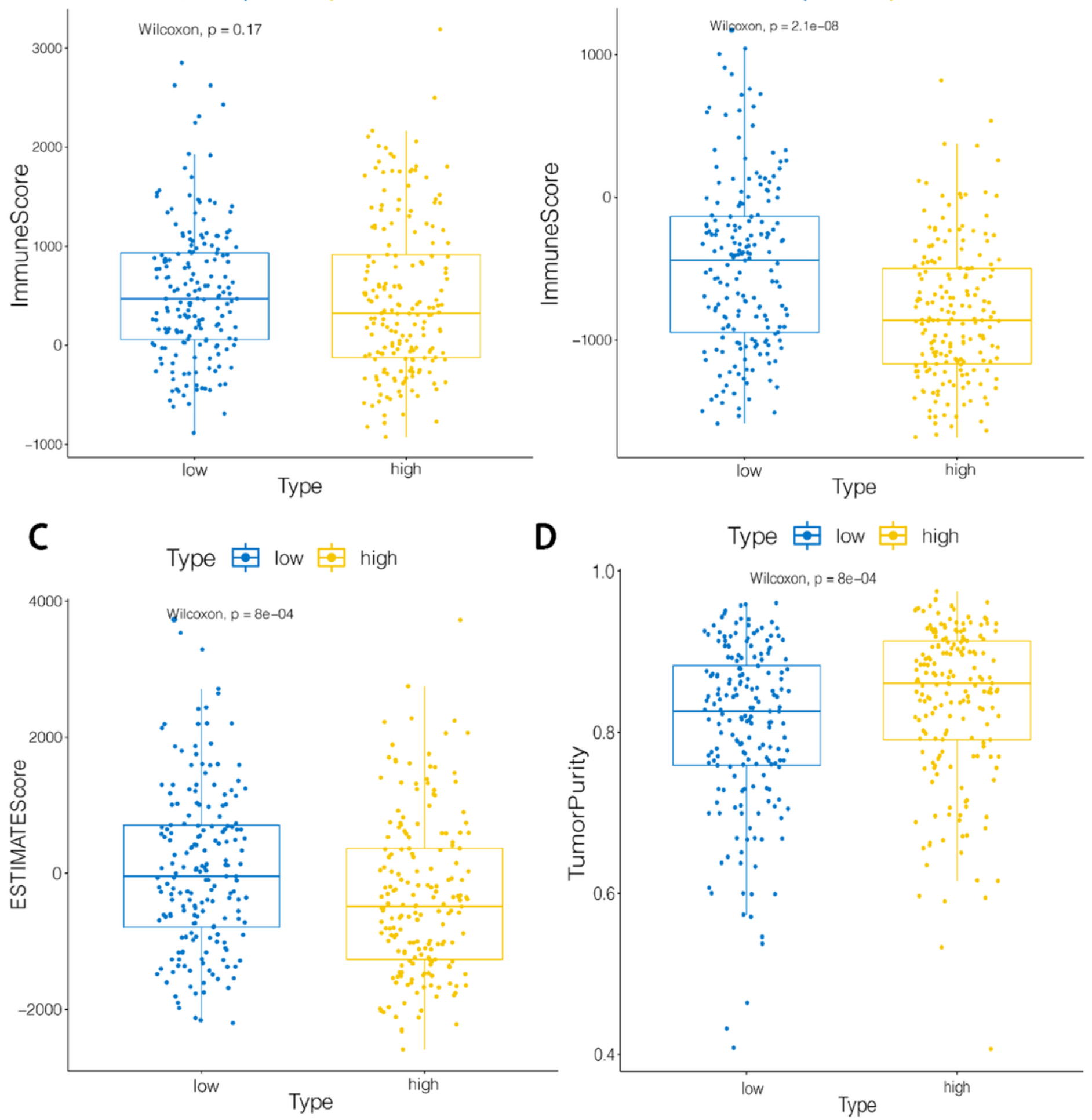

\section{Figure 4}

(A) Box diagram showing the immune score in the high- and low-SNRPC expression groups. (B) Box diagram showing the stromal score in the high- and low-SNRPC expression groups. (C) Box diagram showing the ESTIMATE score in the high- and low-SNRCP expression groups. (D) Box diagram showed the tumor purity in the high- and low-SNRPC expression groups. 
A

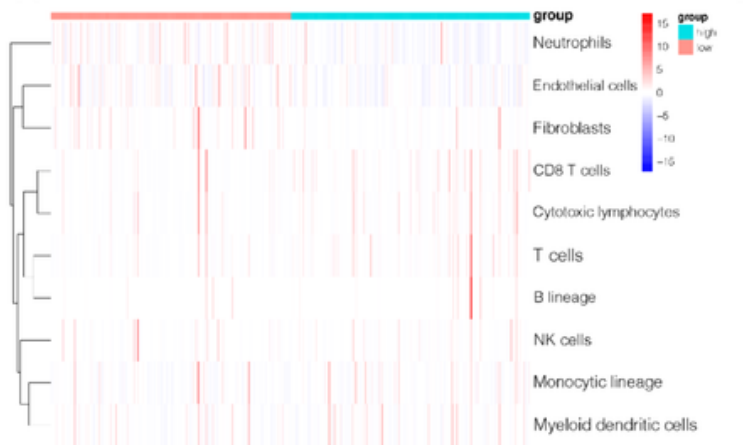

B

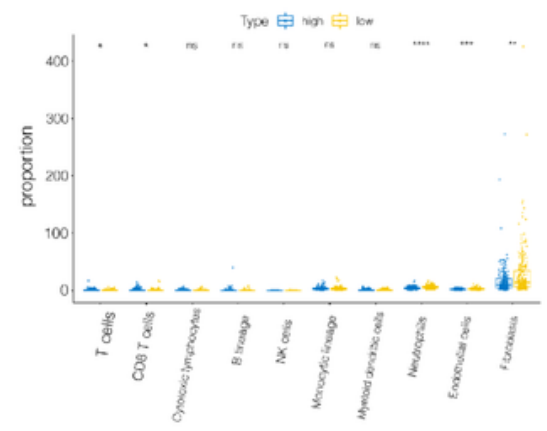

C

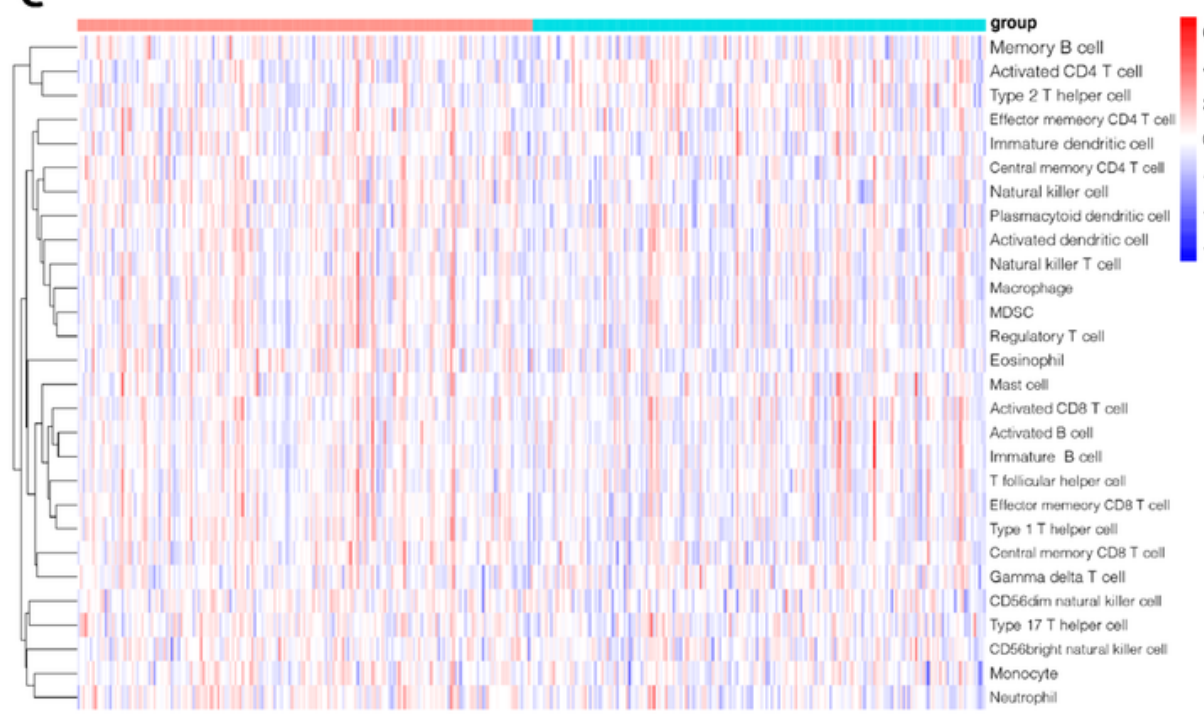

D

Type 官 high 官 low

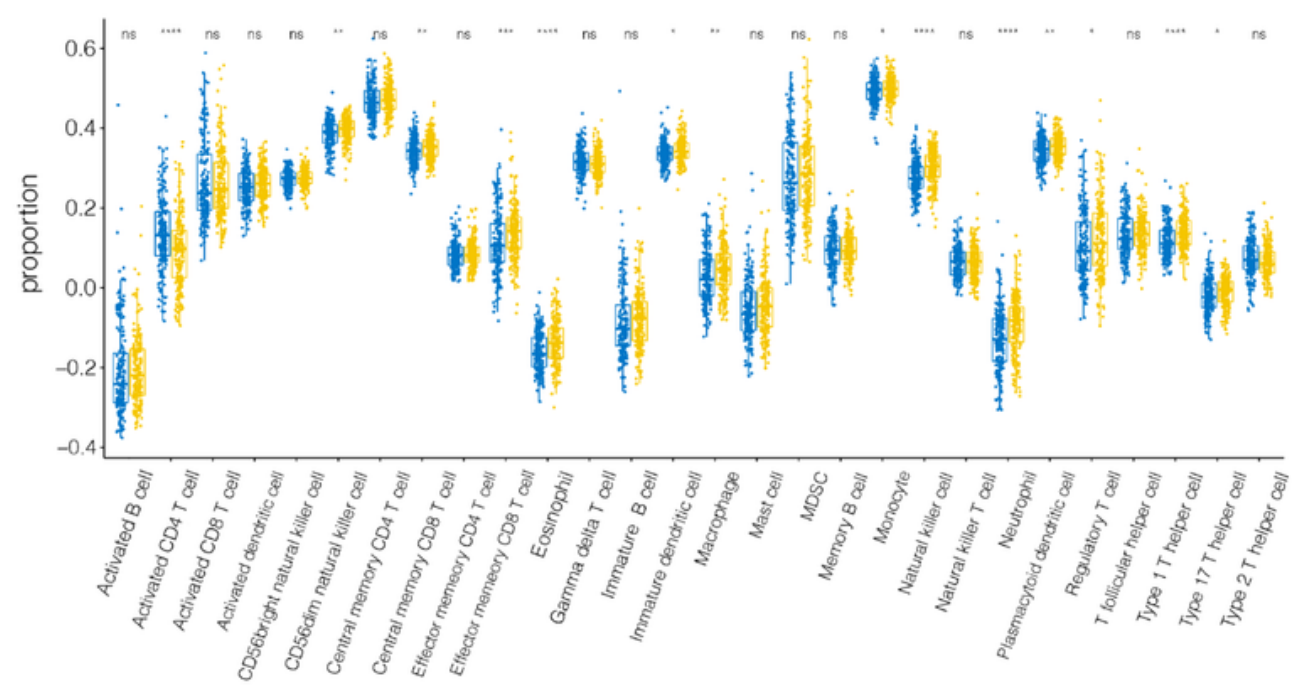

Figure 5

(A) The abundance of TME cells between high- and low-SNRPC expression groups was calculated using MCP-counter. (B) Box plot showing the proportion of TME cells between high- and low-SNRPC expression groups analyzed by MCP-counter. (C, D) Heat map and box plot showing the infiltration of immune cells between high- and low-SNRPC expression groups calculated by sSGSEA. 


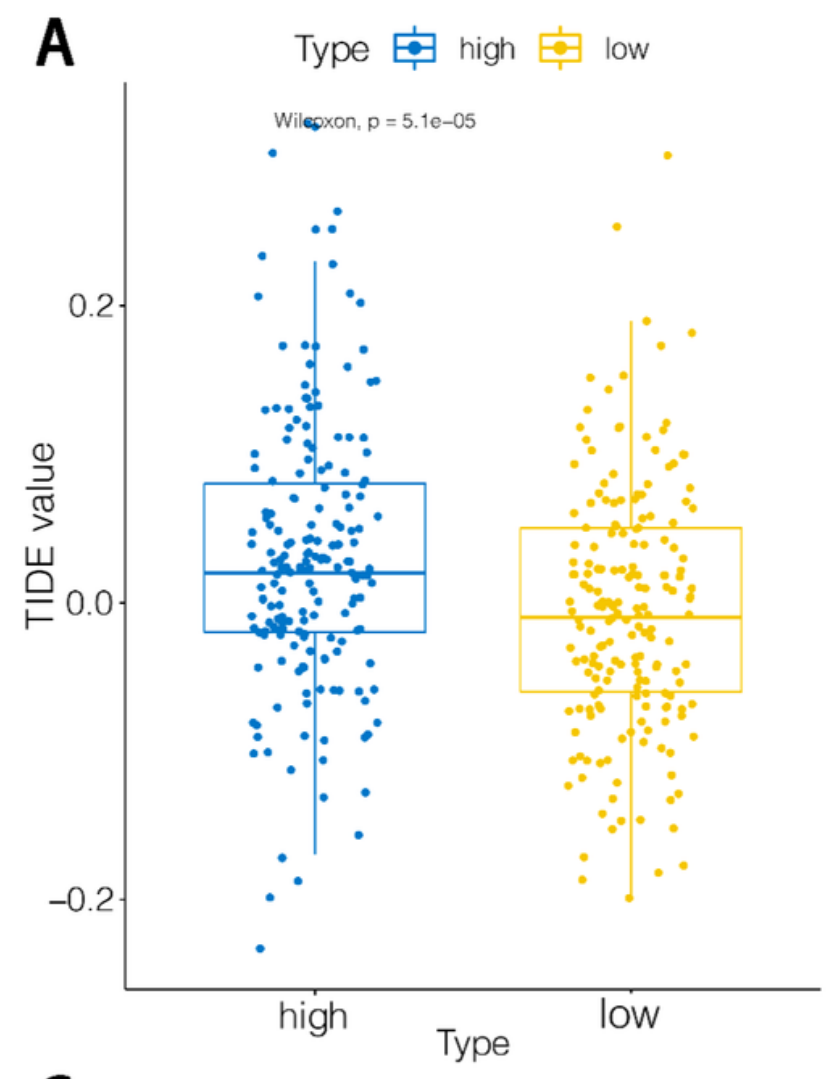

C

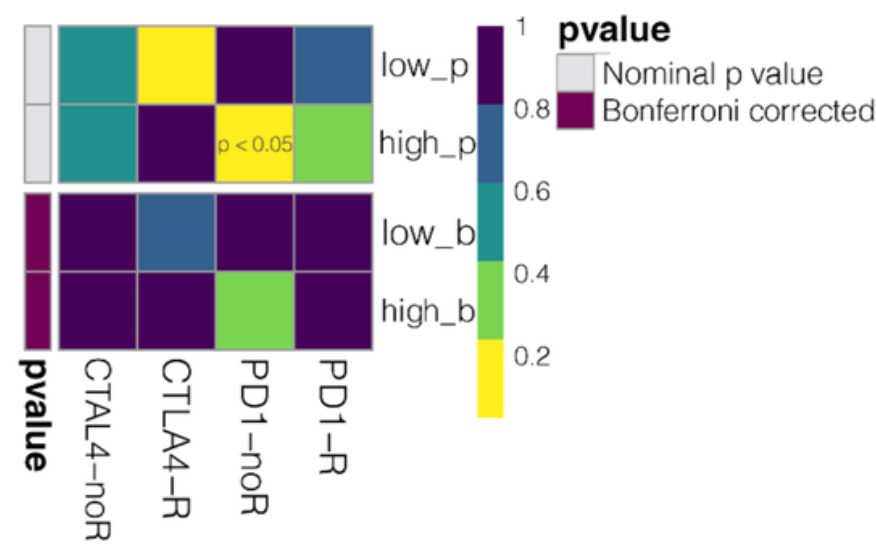

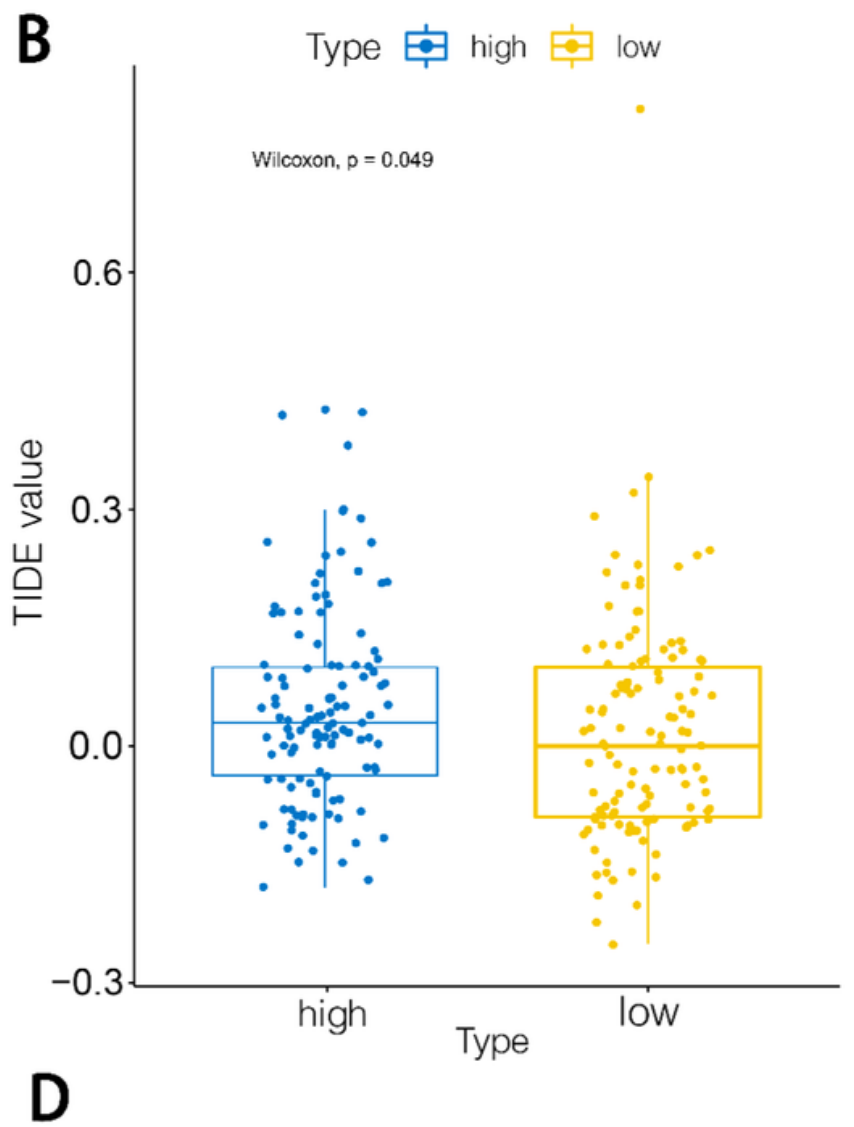

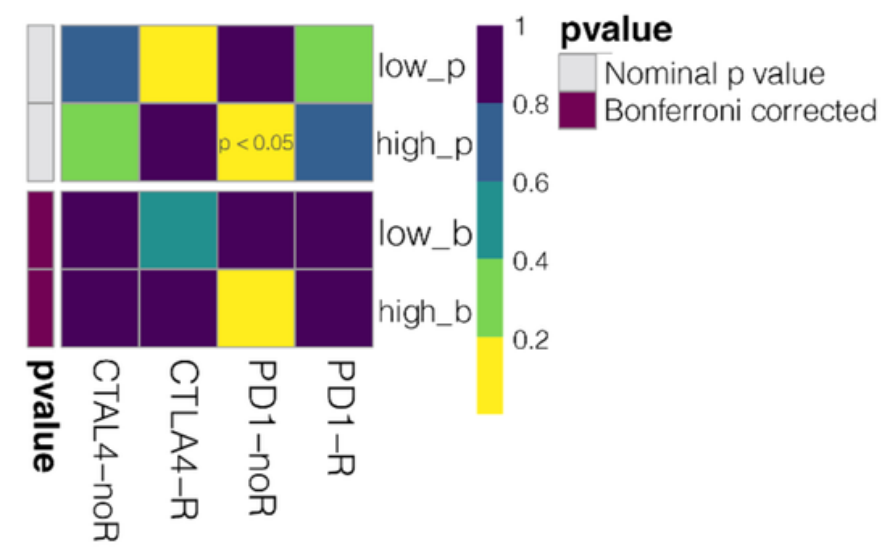

\section{Figure 6}

(A) TIDE score for high- and low-SNRPC expression groups using samples from TCGA database. (B) TIDE scores of high- and low-SNRPC expression groups using data from the ICGC database. (C) Immunotherapy responses between high- and low-SNRPC expression group in TCGA database samples. (D) Immunotherapy responses between high- and low-SNRPC expression group in the ICGC database samples. 